\title{
Signal detection and localization by real observers'
}

\author{
RAMON L. HERSHMAN, ${ }^{2}$ NAVAL ELECTRONICS LABORATORY CENTER, SAN DIEGO \\ J. R. LEVINE, SAN DIEGO STATE COLLEGE \\ M. LICHTENSTEIN, NAVAL ELECTRONICS LABORATORY CENTER, SAN DIEGO
}

A model for detection and localization (DAL) was applied to the processing of a noise-masked tonal signal by human Ss. The model was supported in that consistent values of $d^{\prime \prime}$ were obtained from rating, forced-choice (FC), and DAL procedures. Also, the rating $d^{\prime}$ accurately predicted per cent correct on the FC and DAL trials. A shortcoming of the theory was its failure to account satisfactorily for the details of $F C$ and $D A \cdot L$ stimulus-response matrices.

In this paper we report tests of a recent model for detection and localization (DAL: Hershman \& Lichtenstein, 1967). In the DAL procedure there are $\mathbf{k}$ noisy observation intervals on each trial with the signal appearing in either none or precisely one of the intervals. The observer (O) makes one of $k+1$ responses, indicating either "no signal present" or "the signal is in interval $j$ " $(1<j<k)$. Thus the task for the $O$ is to detect the signal (if present) and locate it in the proper interval.

Hershman and Lichtenstein obtained the decision rule for the ideal DAL $O$ who seeks to maximize his per cent correct responses. Here it is agreed that the $\mathbf{O}$ is scored "correct" on a signal-plus-noise (SN) trial only if he correctly specifies the observation interval containing the signal; he is scored "correct" on a noise-only $(N)$ trial only if he reports "no signal present." The rule is such that when the $k+1$ trial types are equiprobable, the ideal $O$ sets a common criterion level on the likelihood ratio in each interval and makes a series of simple Yes-No (YN) decisions. If each of the $k$ ratios is below the criterion level, a "no signal present" decision is made; if any ratio is above it, the $O$ then makes a Forced-Choice (FC) decision, locating the signal in that interval having the largest likelihood ratio. ${ }^{3}$ Thus it can be seen that the DAL model is a natural extension of the Theory of Signal Detectability (TSD: Swets, 1964; Green \& Swets, 1966). The efficacy of this extended model is the primary subject of this paper.

For the DAL paradigm, Hershman and Lichtenstein assumed equal-variance Gaussian distributions for a monotonic transformation of the likelihood ratio in both $N$ and $S N$ intervals. They then obtained the relation between per cent correct and the detectability index, $\mathrm{d}^{\prime}$, defined as the normalized separation between the distributions. These results were published in tabular form for eight values of $k$ between 2 and 100 (Hershman \& Small, 1968) and will be used to extract $d^{\prime}$ from our experimentally obtained values of per cent correct. In deriving the tables, it was assumed throughout that the $O$ set his likelihood ratio criterion in the optimum fashion. It is also of interest to treat the more general case where an optimum threshold may not be adopted. Taylor and Fraser (1965) have published such a table of $d^{\prime}$ for the $k=2$ case.

Insofar as the ideal DAL decision rules apply to real Os, the d' extracted from a DAL experiment should agree with that obtained from a rating or FC procedure. The present experiment, involving the acoustic detection of a tonal signal in white noise, sought to test this implication and examine other features of the model as well. An earlier report (Hershman, Lichtenstein, \& Levine, 1967) had found largely negative results in the context of a visual detection problem.

\section{METHOD}

Three students at San Diego State College served together as paid Ss. Each trial started with a warning period of white noise that preceded the first observation interval by $1.0 \mathrm{sec}$ and terminated $0.3 \mathrm{sec}$ after the last observation interval. Each observation interval was marked by a $0.5-\mathrm{sec}$ light flash so that whenever a signal occurred it was synchronous with the flash. A 4.0-sec response period followed the last observation interval. The $\mathbf{S}$ wrote his response in an answer booklet.

There were three kinds of trials-rating, FC, and DAL. On rating trials there was only one observation interval so that a new trial started every $5.5 \mathrm{sec}$. The probability of an $\mathrm{N}$ (or SN) presentation was 0.5 . The Ss were instructed to use an integer from 1 to 5 to indicate their confidence that a signal was or was not presented; a response of " 5 " indicated extreme confidence of signal presence.

On three-alternative Forced-Choice (3AFC) trials, there was $1.5 \mathrm{sec}$ between the onset of successive listening intervals so that a new FC trial started every $9.5 \mathrm{sec}$. The signal appeared in precisely one of the three intervals, with $P(S N)=1 / 3$ for each. The $S$ selected one of the three intervals as containing the signal by circling the numeral " 1 ," " 2 ," or " 3 " on his answer sheet.

On DAL trials there were two listening intervals with $1.5 \mathrm{sec}$ between their onsets. Thus, a new DAL trial started every 7.5 sec. Here there were three equiprobable trial types: signal in the first interval only, signal in the second interval only, and signal in neither interval. The $\mathrm{S}$ made his choice from among these alternatives by circling " 1 ," " 2 ," or "neither" on his answer sheet.

The signal was a "pure" tone of $1000 \mathrm{cps}$, generated by a Hewlett-Packard audio oscillator (Model 201CR). The white noise was generated by a Naval Electronics Laboratory thermal noise generator (Model 5834). The signal and noise were mixed electronically and then presented over a binaural headset (Koss, Model PRO-600).

The Ss served in 10 daily sessions of approximately $2 \mathrm{~h}$ each. The first 5 days were used for practice and to adjust signal and noise to yield $50-60 \%$ correct for the FC trials. The last 5 days were devoted to testing, which included 2880 trials for each S. There were 960 trials each of rating, FC, and DAL, given in blocks of 60 trials of each type. An equal number of blocks of each procedure were interleaved each day, with a 2 -min rest period separating each block.

The Ss were informed of the effective a priori probability structure at the outset of the experiment, and they were allowed to score their first block of trials for the first 3 days. After that, they received no feedback as to the accuracy of their decisions. However, Ss were given a preview of the 1000 cps signal at the start of alternate blocks during each session.

\section{Consistency of $\mathbf{d}^{\prime}$}

\section{RESULTS}

First, estimates of $d^{\prime}$ were obtained for each $S$ for each procedure. For the rating trials, the five categories were collapsed to yield a $\mathrm{YN}$ experiment by treating responses of " 4 " and " 5 " as "yes," treating responses of " 1 " and " 2 " as "no," and splitting the " 3 " responses equally. The tables given by Elliott (1964) were then entered to obtain $d^{\prime}$. For the 3 AFC trials, we recovered $d^{\prime}$ from the approximation reported by Elliott $(1964$, p. 680$)$, and for the DAL trials we used the tables of Hershman and Small (1968). The three d's for each S 
Table 1

Extracted Values of d' for Each Procedure

\begin{tabular}{ccccc}
\hline Subject & Rating & FC & DAL & DAL $^{b}$ \\
\hline S1 & 0.51 & 0.53 & 0.69 & 0.77 \\
S2 & 1.00 & 0.97 & 0.95 & 1.11 \\
S3 & 1.06 & 0.92 & 1.08 & 1.09
\end{tabular}

a Assumes coptimum threshold selection, Uershman \& Small (1968)

$b$ provides for non-optimum threshold, Taylor \& Fraser (1965)

appear in Columns 2-4 of Table 1. These extracted indices are quite uniform and serve to reinforce the model at this level of prediction. (The entries in the rightmost column of Table 1 will be discussed in a later section of the paper.)

\section{Predicting Per Cent Correct}

Next, we used the extracted $d^{\prime}$ from the rating trials to predict performance on the FC and DAL trials. Figure I shows the predicted and observed per cent correct for each $S$. Chi-squares were computed on the associated frequencies and summed across the three Ss. These failed to reach the 0.05 significance level for either the FC trials $\left(\chi^{2}=6.88, \mathrm{df}=3\right)$ or the DAL trials $\left(x^{2}=7.23\right.$. $\left.\mathrm{df}=3\right)$. Thus the extended TSD model permits quite satisfactory prediction of per cent correct across the three psychophysical procedures.

\section{FC vs DAL Performance}

On the basis of numerical results, Hershman and Lichtenstein (1967) conjectured that for the ideal $O$ the $(k+1) A F C$ problem is easier-that is, it yields higher per cent correct-than the DAL problem with $k$ observation intervals. Taylor (1968) has recently given a proof of this conjecture based on the number of bilateral contrasts in the two procedures.

The following argument is also compelling. (Here we will use $\mathrm{DAL}_{k}$ to denote the DAL problem with $k$ observation intervals.) First, notice that the $Y N$ problem is identical to $D A L_{1}$, the one-observation DAL problem. Second, we use the result that the $2 \mathrm{AFC}$ problem is easier than the $\mathrm{YN}$ problem; the detectabilities are $\sqrt{2} d^{\prime}$ and $d^{\prime}$, respectively (e.g., Green \& Swets, 1966, p. 68 ). We combine these two stipulations to yield the result that $2 A F C$ is easier than $D_{A L}$. Finally, we can safely add any number of observation intervals, say $\mathrm{k}-1$, to each of these problems without affecting their relative order of difficulty. This yields the desired result that $(k+1) A F C$ is easier than $\mathrm{DAL}_{\mathbf{k}}$.

An alternative line of reasoning ${ }^{4}$ also leads to the same result, and we offer it here because of its intuitive appeal. For this purpose, note that the a priori signal structure and the trial-by-trial stimulus events in the $(k+1) A F C$ and $D A L_{k}$ problems are identical except that the DAL $O$ is denied the information from one of the intervals. (His "no signal present" decision corresponds to the decision that the signal is in the unseen interval.) It follows that the $\mathrm{FC} \mathrm{O}$, who is exposed to all the observation intervals, will perform better.

Here we have limited data that is relevant to this prediction for $k=2$. Going from $D L_{2}$ to $3 A F C$, the rating $d^{\prime}$ predicts small theoretical increases in proportion correct: $0.03,0.06$, and 0.06 for Ss 1, 2, and 3, respectively. For each $S$, a $95 \%$ confidence interval for the difference between his two test proportions was constructed around his expected increase; the number of trials was 960 . The observed changes were -0.01 , 0.06 , and 0.02 (thus a decrease for $S 1$ ). Subject 2 matched the predicted improvement, and the small reversal by $S 1$ lay within his confidence interval. The observed improvement by $\mathrm{S} 3$ was significantly less than the model predicted.

\section{Predicting the Stimulus-Response Matrices}

In pursuit of more detailed predictions, we next used the rating $d^{\prime}$ to predict each term of each S's DAL stimulus-response matrix, that is, the probability of his responding "first," "second," or "neither" given each of the three kinds of trials. These predictions follow from the ideal- $O$ model and are given below.

Let $t_{1}, t_{2}$, and $t$ denote the three DAL trial types-signal in the first interval, signal in the second interval, and signal in neither interval; let $r_{1}, r_{2}$, and $r$ denote the corresponding three responses-."signal in the first interval," "signal in the second interval," and "signal in neither interval." We seek the probability $P\left(r_{j} \mid t_{j}\right)$ that the ideal $O$ makes the row response $r_{j}$ given the column trial type $t_{i}$. As we have noted earlier, in the equal a priori case the optimum DAL decision rule is to first make $k$ Yes-No (that is, threshold) decisions and then follow with an FC decision if any of the likelihood ratios is suprathreshold. ${ }^{3}$ Applying this rule to the assumption of normally distributed, unit-variance inputs with means at 0 and $d^{\prime}$ yields a threshold at $d^{\prime} / 2$; accordingly, the $O$ correctly responds "no signal present" on a noise-only trial with the probability that both noise inputs are less than $d^{\prime} / 2$. If we let $F$ be the cumulative normal function, then

$$
P\left(r_{-} \mid t_{-}\right)=\left[F\left(d^{\prime} / 2\right)\right]^{2} .
$$

Given a $t$ trial, the $O$ makes the (incorrect) responses $r_{1}$ and $r_{2}$ with equal probability so that

$$
P\left(r_{1} \mid t_{-}\right)=P\left(r_{2} \mid t_{-}\right)=1 / 2\left\{1.0-\left[F\left(d^{\prime} / 2\right)\right]^{2}\right\} \text {. }
$$

Thus tirese three entries of the matrix are readily available from tables of the normal function.

We next note that the signal is equally detectable in either interval so we have

$$
\begin{aligned}
P\left(r_{1} \mid t_{1}\right)=P\left(r_{2} \mid t_{2}\right) & =F\left(d^{\prime} / 2\right)\left[1.0-F\left(-d^{\prime} / 2\right)\right]+V \\
& =\left[F\left(d^{\prime} / 2\right)\right]^{2}+V
\end{aligned}
$$

where

$$
\begin{aligned}
& V=\int_{d^{\prime} / 2}^{\infty} \int_{z}^{\infty}\left\{(2 \pi)^{-1 / 2}\right. \exp \left[-1 / 2\left(y-d^{\prime}\right)^{2}\right] \\
&\left.(2 \pi)^{-1 / 2} \exp \left(-1 / 2 z^{2}\right)\right\} d y d z
\end{aligned}
$$

The product to the left of the plus sign in Eq. 3 is the

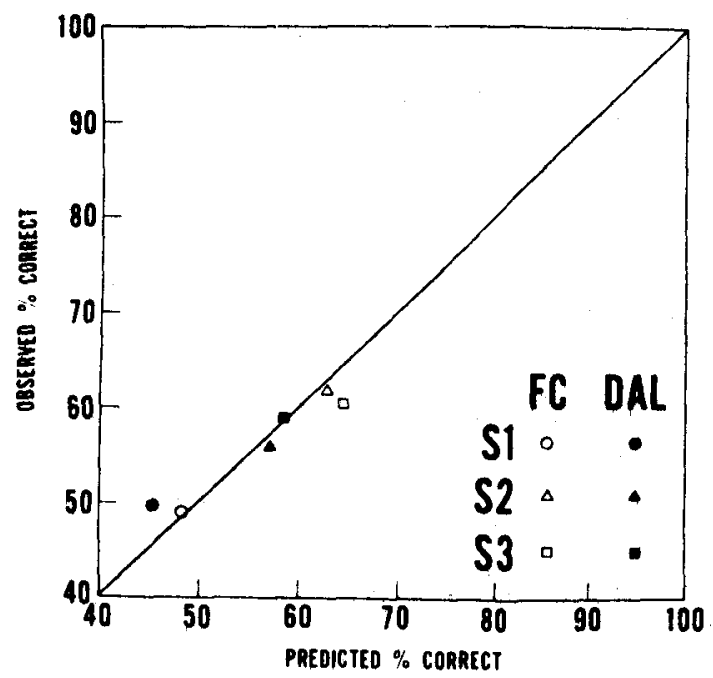

Fig. 1. Observed and predicted per cent correct for the DAL and FC trials; predictions are based on each S's rating d'. 
probability that the signal-plus-noise input will be suprathreshold and that the noise-alone input will be subthreshold. The other cases of correct detection occur when both inputs are suprathreshold and the signal-plus-noise input is larger. These latter are included in the term $V$ which is the probability that a variate $y$ from a normal $\left(d^{\prime}, 1\right)$ distribution and a variate $z$ from a normal $(0,1)$ distribution will both exceed $d^{\prime} / 2$ and that $y>z$. We have programmed a digital computer to generate the cell entries given in $\mathrm{Eq} .3$.

The terms $P\left(r_{-} \mid t_{1}\right)$ and $P\left(r_{-} \mid t_{2}\right)$ are also available from tables of the normal function. Each of these is the probability that a noise-only input and a signal-plus-noise input are both subthreshold, so that

$$
P\left(r_{-} \mid t_{1}\right)=P\left(r_{-} \mid t_{2}\right)=F\left(d^{\prime} / 2\right) F\left(-d^{\prime} / 2\right) .
$$

The remaining terms of the matrix are then obtained by subtraction, that is,

$$
\begin{aligned}
P\left(r_{2} \mid t_{1}\right)=P\left(r_{1} \mid t_{2}\right) & =1.0-P\left(r_{1} \mid t_{1}\right)-P\left(r_{-} \mid t_{1}\right) \\
& =1.0-P\left(r_{2} \mid t_{2}\right)-P\left(r_{-} \mid t_{2}\right) .
\end{aligned}
$$

It is much more straightforward to make matrix predictions for the FC trials. Here we again use the rating d' to obtain $P(C)$ from Elliott's approximation and then fill the'nine cells by symmetry. In the FC matrix, we let $t_{3}$ denote a signal presentation in the third interval and $r_{3}$ denote a "third interval" response.

\section{Matrix Results}

Table 2 shows the observed and theoretical matrices for each S. Two entries appear in each cell: the observed and the

Table 2

Obnerved and Predicted Stimulu-Rerpone Matrices for the DAL and FC Problenu; Predictions Appen in Parentheses and are Band on each S's Raties d'; Vslues of chi-quare Appear Bdow esch Matrix

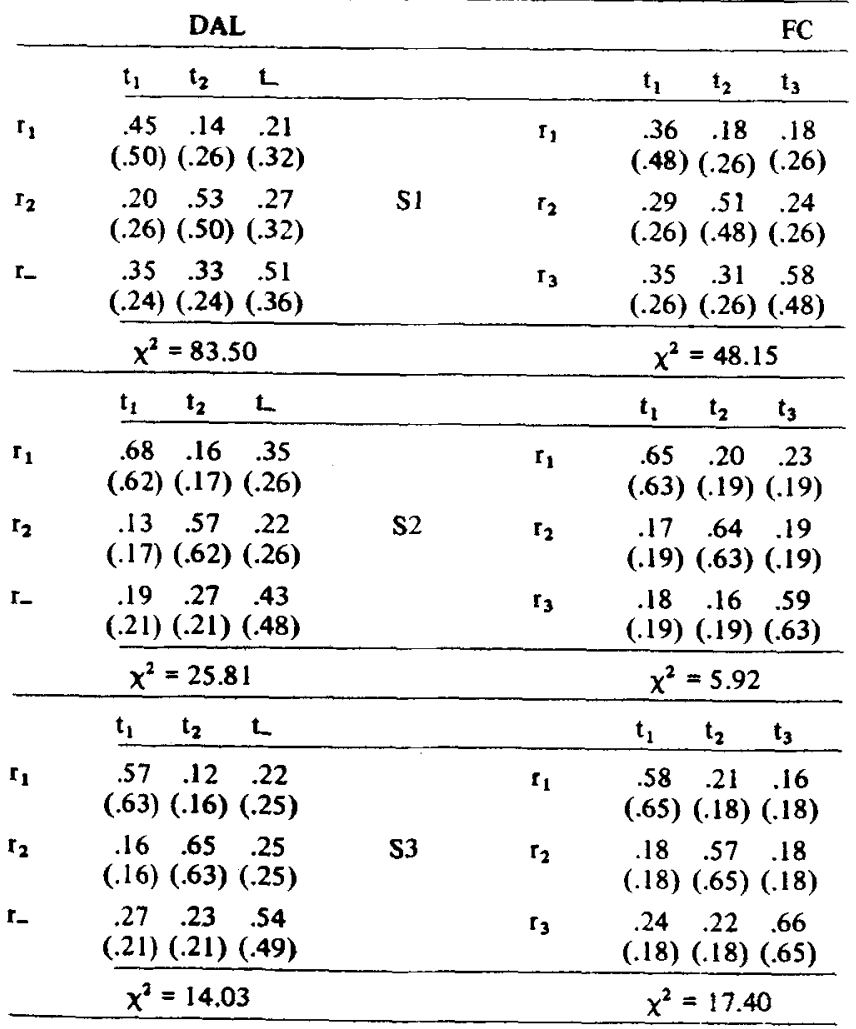

Table 3

General DAL Stimulus-Response Matrix for the Equal a priori Experiment

\begin{tabular}{ccccc}
\hline$r_{1}$ & $t_{1}$ & $t_{2}$ & $t$ & \\
$r_{2}$ & $a$ & $b$ & $\frac{1-c}{2}$ & $\Sigma=1+e$ \\
$r_{-}$ & $b$ & $a$ & $\frac{1-c}{2}$ & $\Sigma=1+e$ \\
\hline
\end{tabular}

predicted (in parentheses) proportion of row responses to the column trial type. Chi-squares were computed on the associated frequencies, and the values of these test statistics also appear in the table. The reader may wish to reconstruct the frequency matrix so we note that the number $n$ of trials of each type were: for the DAL trials, $n\left(t_{1}\right)=300, n\left(t_{2}\right)=358$, $n\left(t_{-}\right)=302$; for the FC trials, $n\left(t_{1}\right)=304, n\left(t_{2}\right)=298$, $n\left(t_{3}\right)=358$.

Although the eyeball fits of Table 2 are reasonably good, each $x^{2}$ was significant $(p<.05$, df $=6)$ except that for the FC matrix of $S 2$. It is clear that $S 1$ is the most contrary of the Os, exhibiting a distinct preference for the third categories, $r_{-}$and $r_{3}$. It is also clear that, in general, the model fails to predict adequately the fine-grain structure of either the DAL or FC matrices.

\section{Performance on Signal vs No-Signal Trials}

Subtracting Eq. 1 from Eq. 3 and noting that the term $V$ is positive, it follows immediately that the ideal DAL $O$ has a higher per cent correct on signal trials $\left(t_{1}\right.$ or $\left.t_{2}\right)$ than on no-signal trials $\left(t_{-}\right)$. From the DAL matrices of Table 2 it can be seen that $S 2$ and $S 3$ do perform better on the signal trials. However, $S 1$ has a higher per cent correct on $t$ trials, due perhaps to his response bias that we have already noted.

\section{Response Bias in the DAL Procedure}

It also can be shown that the ideal DAL O has a "bias" for saying "signal present." Of course, this is not a whimsical preference, but we use the word "bias" here to refer to the fact that the theoretical $O$ does not split his responses equally even though the three trial types are equiprobable.

Consider the general equal a priori DAL matrix of Table 3. Here we have used the symmetry of the problem to write $P\left(r_{1} \mid t_{1}\right)=P\left(r_{2} \mid t_{2}\right)=a ; P\left(r_{2} \mid t_{1}\right)=P\left(r_{1} \mid t_{2}\right)=b$, etc. We first assert that $(1-c) / 2>1-a-b$, that is,

$$
P\left(r_{1} \mid t_{-}\right)=P\left(r_{2} \mid t_{-}\right)>P\left(r_{-} \mid t_{1}\right)=P\left(r_{-} \mid t_{2}\right) \text {. }
$$

To prove this, we argue that $P\left(r_{1} \mid t_{-}\right)$is the sum of two components: (1) the probability that the first noise input is suprathreshold and the second one is subthreshold, and (2) the probability that both noise inputs are suprathreshold with the first one being larger. But Component (1) is given by $F\left(d^{\prime} / 2\right) \cdot F\left(-d^{\prime} / 2\right)$, which is precisely $P\left(r_{-} \mid t_{1}\right)$ of Eq. 5 . Component (2) is certainly positive, so the inequality of Eq. 7 follows. Thus we can write

$$
(1-c) / 2=(1-a-b)+e, \quad e>0 .
$$

Using Eq. 8 and summing the probabilities in each row, we obtain

$$
P\left(r_{1}\right)=P\left(r_{2}\right)=a+b+(1-a-b)+e=1+e .
$$

Thus the receiver's bias for responding "signal present" is equal to $2 \mathrm{e}$ where $e$ is the difference between the off-diagonal entries of the third row and third column. It is possible to obtain a direct expression for the bias $2 \mathrm{e}$. We have that 


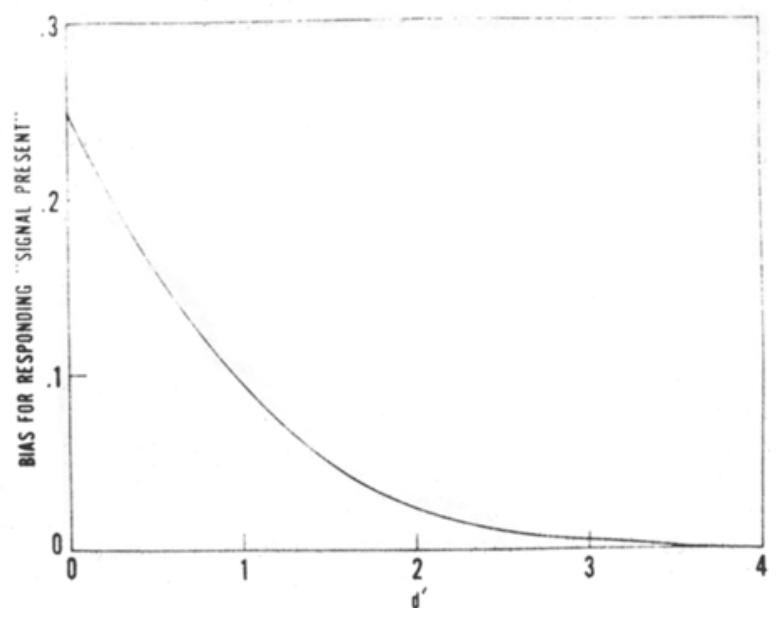

Fig. 2. Response bias of the ideal DAL $O$ as a function of $d^{\prime}$

$$
\begin{aligned}
2 e & =\left[P\left(r_{1} \mid t_{-}\right)-P\left(r_{-} \mid t_{1}\right)\right]+\left[P\left(r_{2} \mid t_{-}\right)-P\left(r_{-} \mid t_{2}\right)\right] \\
& =\left[P\left(r_{1} \mid t_{-}\right)+P\left(r_{2} \mid t_{-}\right)\right]-\left[P\left(r_{-} \mid t_{1}\right)+P\left(r_{-} \mid t_{2}\right)\right] .
\end{aligned}
$$

Substitution from Eqs. 2 and 5 yields

$$
2 e=1.0-\left[F\left(d^{\prime} / 2\right)\right]^{2}-2 F\left(d^{\prime} / 2\right) F\left(-d^{\prime} / 2\right)
$$

Using the relation $F(x)=1.0-F(-x)$, we have

$$
\begin{aligned}
2 e & =1.0-\left[F\left(d^{\prime} / 2\right)\right]^{2}-2 F\left(d^{\prime} / 2\right)\left[1.0-F\left(d^{\prime} / 2\right)\right] \\
& =1.0-2 F\left(d^{\prime} / 2\right)+\left[F\left(d^{\prime} / 2\right)\right]^{2} \\
& =\left[1.0-F\left(d^{\prime} / 2\right)\right]^{2}
\end{aligned}
$$

The amount of bias given by Eq. 12 is plotted as a function of $d^{\prime}$ in Fig. 2. Thus with stronger signals the theoretical receiver's "bias" tends to vanish and his response output more closely matches the a priori schedule of $P($ signal $)=2 / 3$, $P($ noise only $)=1 / 3$.

We next compared the predicted DAL response biases with those exhibited by our experimental Ss. The rating d' was used together with the result of Eq. 12 to form the predictions. These observed and predicted (in parentheses) proportions of "signal" responses were as follows: $S 1=0.607(0.722)$; S $2=0.706(0.704) ; \quad S 3=0.661(0.702)$. Chi-squares were computed on the associated frequencies, and these statistics were significant $(p<.05, \mathrm{df}=1$ ) for $S 1$ and $S 3$. Thus $S 1$ had a clear bias for saying "neither" which was opposite to that of the theoretical bias. Subject 2 matched the predicted bias, while $S 3$, contrary to the theory, exhibited no bias whatever.

\section{DISCUSSION}

On balance, we find the DAL formulation quite tenable as a model for our Os. Most importantly, it leads to the extraction of detectability indices that are consistent with those obtained from the rating and $\mathrm{FC}$ procedures. A related result is that one can use the $\mathrm{d}^{\prime}$ index from the rating trials to satisfactorily predict the percentage of correct DAL responses. In this sense, then, there is reinforcement for the notion that the extended TSD model may be brought to bear directly on the DAL task. In turn, this task is more akin to many real-world detection problems than are the familiar $\mathrm{YN}$ and FC paradigms.

On the other hand, the model is inadequate when pressed to predict the detailed structure of the DAL stimulus-response matrices. Indeed the more hasic TSI) formulation fails to predict such matrices for the standard $F($ experiment. although the more gross measure of per cent correct is again readily predictable.

One possible reason for the failure at detailed $D \wedge L$ prediction is that Os do not (as we have assumed they do) set an optimum criterion level in the DAL situation. We can pursue this hypothesis in that the paper of Taylor and Fraser (1965) makes it possible to extract d' from DAL data without committing oneself to the notion of selection of an optimum criterion level. Their computer algorithm considers only those trials on which a signal was in fact presented. It converges to that $\mathrm{d}^{\prime}$ and threshold setting associated with (1) the observed proportion of correct responses on signal trials and (2) the observed proportion of wrong responses on such trials (wrong here does not include the "neither" responses). The value of $\mathrm{d}^{\prime}$, but not the criterion level, is made available in the Taylor and Fraser table which assumes equal standard deviations for the two distributions. 5

We applied the Taylor and Fraser results to the observed DAL matrices of Table 2 and obtained the $d^{\prime}$ values shown in Column 5 of Table 1. These entries should be compared with the DAL d's for which we assumed an optimum criterion level (Column 4 of Table 1). For S 1 and S 2 these lat ter indices are smaller. We might then be tempted to conclude that indeed these Ss failed to maximally exploit their available d's, that is, they degraded them with a nonoptimum threshold. On this basis we should also conclude that S 3 was using a correct criterion level; however, this certainly conflicts with our inadequate prediction of his DAL S-R matrix. Thus it seems that the present discrepancies between theory and data are not resolved by the straightforward introduction of a faulty criterion mechanism.

We also point out in Table 1 that our DAL d's of Column 4 are more consistent with the rating and $F C$ indices than are those derived from the nonoptimum criterion notion. This is somewhat puzzling, for we should expect that providing for a nonoptimum criterion setter would sharpen the correspondence among d's from the several procedures. We are not able to resolve this difficulty here but can only remark that our $d^{\prime}$ estimates, which are based on all DAL trials, seem to yield more consistent indices than estimates derived only from the signal-present trials.

Finally, the failure to accurately predict the FC matrices from the rating $d$ 's is noteworthy. Thus, the observed invariance of $\mathrm{d}^{\prime}$ across experimental procedures (see the data summarized by Green \& Swets, 1966, p. $110 \mathrm{ff}$ may well be misleading with respect to the generality of the TSD model. We strongly recommend that other investigators include in their repertoires the goodness-of-fit test for S-R matrices.

\section{REFERENCES}

ELLIOTT, P. B. Tables of $\mathrm{d}^{\prime}$. In J. A. Swets (Ed.), Signal detection and recognition by human obsenvers. New York: Wiley, 1964.

GREEN, D. M., \& SWETS, J. A. Signal detection theory and psychophysics. New York: Wiley, 1966.

HERSHMAN, R. L., \& LICHTENSTEIN, M. Detection and localization: An extension of the theory of signal detectability. Journal of the Acoustical Society of America, 1967, 42, 446-452.

HERSHMAN, R. L., \& SMALL, D. Tables of $\mathrm{d}^{\prime}$ for detection and localization. Perception \& Psychophysics, 1968, 3, 321-323.

HERSHMAN, R. L., LICHTENSTEIN, M., \& LEVINE, J. R. An ideal observer for detection and localization (DAL). Paper presented at the meeting of the Psy chonomic Society, Chicago, Illinois, October, 1967.

SWETS, J. A. (Ed.), Signal detection and recognition by human obsenvers. New York: Wiley, 1964.

TAYLOR, M. M. Detection and localization experiment: Comments on a paper by Hershman and Lichtenstein and a note on a table of $d^{\prime}$ for $k=2$. Journal of the Acoustical Society of America, 1968, 43, 884-885. 
TAYLOR, M. M., \& FRASER, W. C. G. A table of $d^{\prime}$ for a model of the unforced choice experiment. Defence Research Medical Laboratories, Report No. 534.

\section{NOTES}

1. A portion of this paper was presented at the meeting of the Acoustical Society of America, Miami Beach, November, 1967. We thank W. A. Hillix for his helpful review of the manuscript.

2. Address: Naval Electronics Laboratory Center, 271 Catalina Blvd., San Diego, California 92152.
3. It can easily be shown that the order of these decisions can be reversed-that is, first $\mathrm{FC}$ and then $\mathrm{YN}$-without changing the receiver's output.

4. We thank $C$. Neuse for this argument.

5. While the Taylor and Fraser algorithm is general in that it finds d's for arbitrary assumptions about the standard deviations, their published table is for the equal sigma case.

(Accepted for publication January 5, 1969.) 